\title{
Focused Ion Beam as a direct-write mask tool for patterning diamond
}

\author{
W.R. McKenzie,* J.B. Pethica* and G.L.W. Cross* \\ *Centre for Research on Adaptive Nanostructures and Nanodevices (CRANN), Trinity College \\ Dublin, Dublin 2, Ireland.
}

Diamond is the ultimate material for a vast array of applications since it exhibits a number of the most extreme properties of any known material such as the widest optical bandgap, highest Young's modulus, hardness, thermal conductivity and highest number density of atoms [1]. One reason that diamond is not used extensively in areas such as microelectronics, optoelectronics, photonics and Micro-Electro-Mechanical Systems (MEMS), is the difficulty in fabrication of structures on its surface, particularly if feature sizes are required at the micro or nano-meter scale and over wide areas.

We present a new process for patterning diamond, illustrated in FIG 1. The starting material is a flat diamond surface, as shown in "A". Step 1, involves exposing this surface to a FIB over the area where the feature is to be created, as shown in "B". This ion implantation results in the formation a hard mask from the etch of the diamond surface during Step 2. Step 2 involves applying an oxygen plasma to the surface which sputters the unexposed diamond and leaves the FIB exposed and masked area raised.

Structures created using this technique can be seen in FIG 2. The optical microscope image in "A" demonstrates patterning of complex features over large areas, in this case over areas $\approx 0.5 \mathrm{~mm}$ in dimension. Patterns of this size can be rapidly fabricated due to the low ion dose of $\sim 10^{16}$ ions $/ \mathrm{cm}^{2}$ required to create the masking effect. "B" demonstrates patterning of features more than three orders of magnitude smaller on a polycrystalline diamond thin film. "C" demonstrates the highest resolution features obtained to date, line structures of $90 \mathrm{~nm}$ pitch and 30nm width. FIG 3 "A" shows a bright field Transmission Electron Microscope image of the cross section from a patterned surface with the diamond lattice oriented parallel to a [110] zone axis. The continuity of the Bragg contrast in this image indicates that the diamond retains its crystal structure and contains no local residual stresses which could be expected as an artifact of the $30 \mathrm{kV} \mathrm{Ga}^{+}$ion exposure in Step 1. The high resolution image of the raised area indicated in the inset of "A", shown in FIG 3 "B", also reveals a structure expected of an undamaged diamond crystal containing no disordered, amorphous or graphitic inclusions or other signs of significant ion damage.

The close correlation between the FIB probe diameter and patterned line width, demonstrated in FIG 2 "C", suggests that the probe diameter limits pattern feature size in this instance. We believe that this dimension could be significantly reduced below $10 \mathrm{~nm}$, the smallest dimensions possible for patterning using resist based lithography [2], if the exposure were applied with the most modern commercially available ion optics, currently woth imaging resolutions of $2.4 \mathrm{~nm}$ for a $\mathrm{Ga}^{+} \mathrm{FIB}$ [3].

Further results in this study will include the first images from the newly built CRANN Microscopy Center using installations of the Carl Zeiss "Orion Plus" He-Ion microscope, Orsay Physics "Cobra" high resolution $\mathrm{Ga}^{+}$FIB column, and FEI Titan Transmission Electron Microscope. 
References

[1] A.D. Greentree et al, MaterialsToday 11 (2008) 22.

[2] S. Manako et al., Jap. J. Appl. Phys. 36 (1997) L724.

[3] Carl Zeiss press release, www.smt.zeiss.com, (2008).

[4] Authors would like to thank the ESTEEM program and Oxford Materials for allowing access and making the TEM component of this research possible.

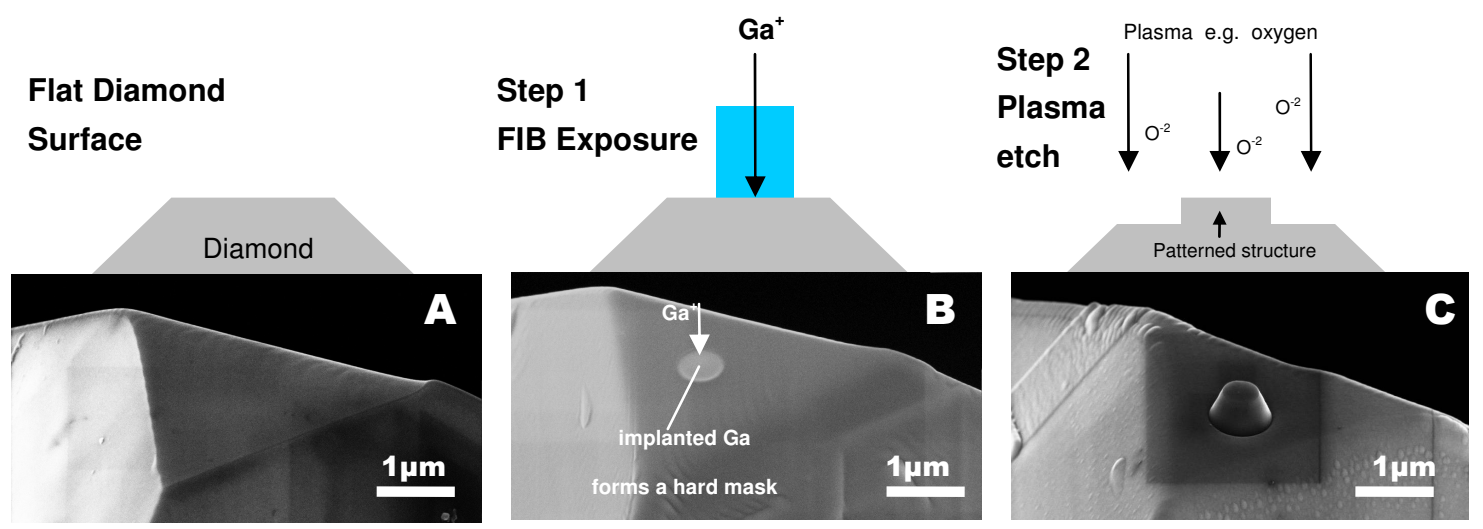

FIG 1. Schematic and corresponding secondary electron SEM images of the diamond fabrication process. Starting with a flat diamond surface "A", Step 1 involves implanting Ga into the surface, as shown in "B", forming a hard mask to the plasma etch of the diamond in Step 2. This plasma etch results in a pattern structure such as that shown in "B".
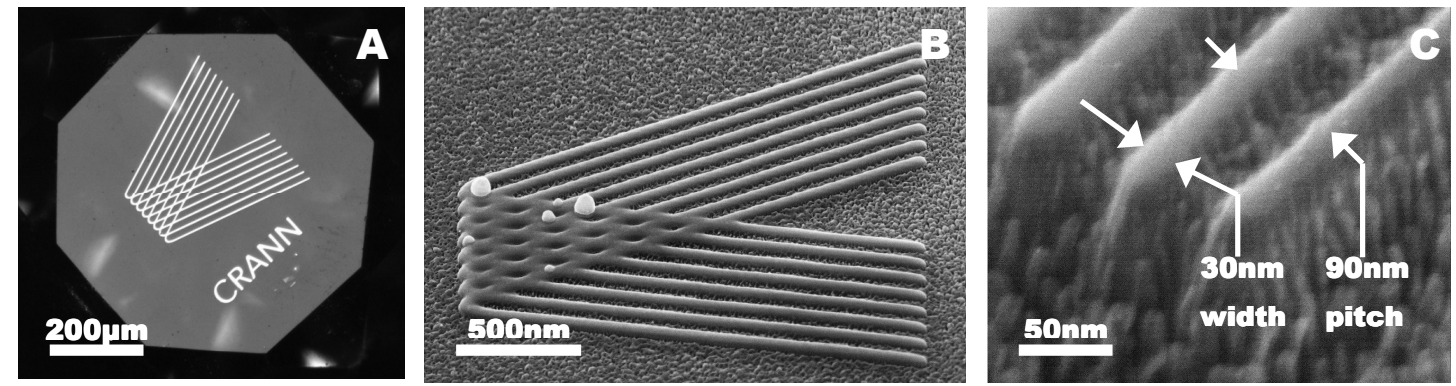

FIG 2. "A" Optical microscope image of a pattern created on the largest facet of a natural diamond cut as an item of jewelry. "B" SEM image of line structures patterned onto a diamond thin film. "C" SEM image of line structures demonstrating a width of $30 \mathrm{~nm}$ and pitch of $90 \mathrm{~nm}$ patterned onto a single crystal diamond.
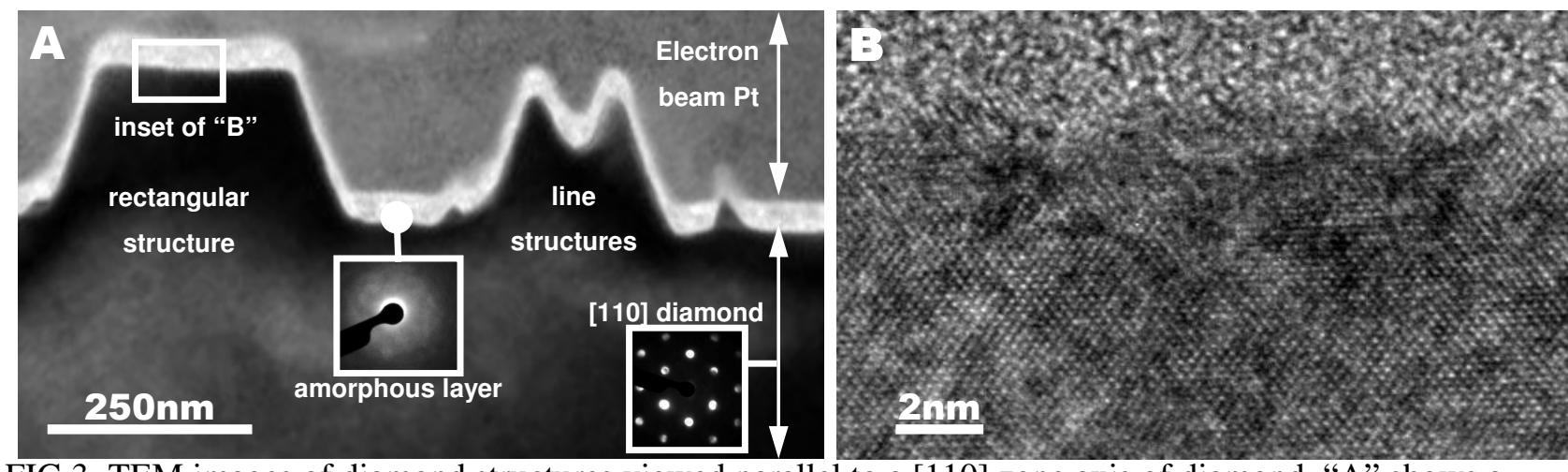

FIG 3. TEM images of diamond structures viewed parallel to a [110] zone axis of diamond. "A" shows a bright field image of patterned rectangular and line structures. Diffraction patterns of the diamond and an amorphous layer are shown as insets. "B" shows a HR-TEM image of the area marked "inset of B". 